\title{
The Graph of Equivalence Classes of Zero Divisors
}

\author{
Vinayak Joshi, B. N. Waphare, and H. Y. Pourali \\ Department of Mathematics, University of Pune, Pune 411007, India \\ Correspondence should be addressed to Vinayak Joshi; vinayakjoshil11@yahoo.com
}

Received 13 December 2013; Accepted 5 February 2014; Published 8 May 2014

Academic Editors: G. Isaak, H.-J. Kreowski, and J. A. Rodriguez Velazquez

Copyright (C) 2014 Vinayak Joshi et al. This is an open access article distributed under the Creative Commons Attribution License, which permits unrestricted use, distribution, and reproduction in any medium, provided the original work is properly cited.

\begin{abstract}
We introduce a graph $G_{E}(L)$ of equivalence classes of zero divisors of a meet semilattice $L$ with 0 . The set of vertices of $G_{E}(L)$ are the equivalence classes of nonzero zero divisors of $L$ and two vertices $[x]$ and $[y]$ are adjacent if and only if $[x] \wedge[y]=[0]$. It is proved that $G_{E}(L)$ is connected and either it contains a cycle of length 3 or $G_{E}(L) \cong K_{2}$. It is known that two Boolean lattices $L_{1}$ and $L_{2}$ have isomorphic zero divisor graphs if and only if $L_{1} \cong L_{2}$. This result is extended to the class of SSC meet semilattices. Finally, we show that Beck's Conjecture is true for $G_{E}(L)$.
\end{abstract}

\section{Introduction}

The idea of a zero divisor graph was introduced by Beck in [1] to investigate the interplay between ring theoretic properties and graph theoretic properties. The concept of zero divisor graph is also well studied in ordered structures; see Alizadeh et al. [2, 3], Halaš and Jukl [4], Halaš and Länger [5], Joshi [6], Joshi et al. [7-11], and $\mathrm{Lu}$ and $\mathrm{Wu}[12]$.

The graph of equivalence classes of zero divisors of commutative rings is well studied in Allen et al. [13], Anderson and LaGrange [14], and Spiroff and Wickham [15].

In this paper, we extend this concept to a meet semilattice $L$ with 0 . The main aspect of the graph $G_{E}(L)$ of equivalence classes of zero divisors of $L$ is the connection to the associated primes of $L$. In general, every vertex of $G_{E}(L)$ either corresponds to an associated prime or is adjacent to an associated prime of $L$. Further, we consider the equivalence relation on a meet semilattice $L$ with $0, x \sim y$ if and only if $\operatorname{ann}(x)=\operatorname{ann}(y)$ for $x, y \in L$ and construct the meet semilattice $L^{\prime}=\left\{[x] \mid x \in Z_{\{0\}}(L)^{*}\right\}$. It is proved that $L^{\prime}$ is an SSC meet semilattice with the property that $G_{E}(L) \cong G_{\{0\}}\left(L^{\prime}\right)$, the zero divisor graph of $L^{\prime}$. Hence it is clear that the study of $G_{E}(L)$ is nothing, but the study of zero divisor graph of SSC meet semilattices. This observation helps us to prove that for bounded SSC meet semilattices $L_{1}$ and $L_{2}, G_{\{0\}}\left(L_{1}\right) \cong$ $G_{\{0\}}\left(L_{2}\right)$ if and only if $L_{1} \cong L_{2}$. This extends the result of LaGrange [16] for Boolean algebra. It is proved that for an SSC meet semilattice $L, G_{\{0\}}(L)$ is connected and either it contains a cycle of length 3 or $G_{\{0\}}(L) \cong K_{2}$. In the class of a finite semicomplemented meet semilattice $L, G_{E}(L)$ is isomorphic to the zero divisor graph $G_{\{0\}}(L)$ if and only if $L$ is SSC.

\section{Properties of the Graph $G_{E}(L)$}

We begin with the necessary definitions and terminology.

A nonempty subset $I$ of a meet semilattice $L$ is said to be semi-ideal, if $y \leqslant x \in I$ implies that $y \in I$. A semi-ideal $I$ of a meet semi-lattice $L$ is said to be an ideal, if $a, b \in I$ implies that $a \vee b \in I$. Dually we have the concept of a filter. A proper semi-ideal (ideal) is said to be prime, if $a \wedge b \in I$ implies either $a \in I$ or $b \in I$.

A prime semi-ideal (ideal) $P$ of a meet semilattice $L$ is said to be minimal prime semi-ideal (ideal), if there is no prime ideal $Q$ such that $Q \varsubsetneqq P$.

The concept of SSC lattices is introduced by Janowitz [17]. We write this definition for a meet semilattice with 0 .

A meet semilattice $L$ with 0 is said to be semicomplemented if, for $a \neq 1$, there exists $b \neq 0$ such that $a \wedge b=0$ and $L$ is said to be section semicomplemented (in brief SSC) if for $a, b \in L$ with $a<b$, there exists nonzero $c$ such that $0<c \leq b$ and $a \wedge c=0$. It is clear that every SSC lattice is a semicomplemented lattice, but not conversely. More details about SSC poset can be found in Thakare et al. [18, 19], Joshi [20].

Let $L$ be a meet semilattice with 0 . The set of all zero divisors of $L$ is denoted by $Z_{\{0\}}(L)=\{x \in L \mid x \wedge y=0$ 




FIGURE 1: The graph $G_{E}(L)$ of equivalence classes of zero divisors.

for some $y \in L \backslash\{0\}\}$ and the set of all nonzero zero divisors is denoted by $Z_{\{0\}}(L)^{*}$. Clearly, $Z_{\{0\}}(L)^{*} \cup\{0\}=Z_{\{0\}}(L)$.

Let $G$ be a graph, and let $x, y$ be distinct vertices in $G$. We denote by $d(x, y)$ the length of a shortest path from $x$ to $y$ if it exists and put $d(x, y)=\infty$ if no such path exists. The diameter of $G$, denoted by $\operatorname{diam}(G)$, is zero if $G$ is the graph on one vertex and is $\operatorname{diam}(G)=$ $\sup \{d(x, y)$ where $x$ and $y$ are distinct vertices of $G\}$ otherwise. A cycle in a graph $G$ is a path that begins and ends at the same vertex. The girth of $G$, written $\operatorname{girth}(G)$, is the length of the shortest cycle in $G$ (and girth $(G)=\infty$ if $G$ has no cycles). The chromatic number of $G$ is denoted by $\chi(G)$. Thus, $\chi(G)$ is the minimum number of colors which can be assigned to the elements of $L$ such that adjacent elements receive different colors. If this number is not finite, write $\chi(G)=\infty$. A subset $C$ of $G$ is a clique if any two distinct vertices of $C$ are adjacent. If $G$ contains a clique with $n$ elements and every clique has at most $n$ elements then the clique number of $G$ is Clique $(G)=n$. If the sizes of the cliques are not bounded, then Clique $(G)=\infty$. We always have $\chi(G) \geqslant \operatorname{Clique}(G)$.

Joshi [6] introduced the concept of the zero divisor graph of a poset $P$ having the smallest element 0 with respect to an ideal $I$ of $P$. We mention below this definition when the corresponding poset is a meet semilattice and $I=\{0\}$. Note that this definition coincides with the definition given by $\mathrm{Lu}$ and $\mathrm{Wu}[12]$ when $I=\{0\}$.

Definition 1 (Joshi [6], Lu and $\mathrm{Wu}$ [12]). Let $L$ be a semilattice with 0 . We associate a simple undirected graph with $L$, called the zero divisor graph of $L$ with respect to $\{0\}$, denoted by $G_{\{0\}}(L)$, with the set of vertices is $V\left(G_{\{0\}}(L)\right)=Z_{\{0\}}(L)^{*}=$ $\{x \in L \backslash\{0\} \mid x \wedge y=0$ for some $y \in L \backslash\{0\}\}$ and two distinct vertices $x, y$ are adjacent if and only if $x \wedge y=0$.

For any $x, y \in L$, we say that $x \sim y$ if and only if $\operatorname{ann}(x)=$ $\operatorname{ann}(y)$, where $\operatorname{ann}(a)=\{b \mid a \wedge b=0\}$. Note that $\sim$ is an equivalence relation on $L$. Furthermore, if $x_{1} \sim x_{2}$ and $x_{1} \wedge$ $y=0$, then $y \in \operatorname{ann}\left(x_{1}\right)=\operatorname{ann}\left(x_{2}\right)$ and hence $x_{2} \wedge y=0$. We define $[x]$, the equivalence class of $x$, as follows: $[x]=\{z \in$ $L \mid \operatorname{ann}(z)=\operatorname{ann}(x)\}$.

Lemma 2. Let $L$ be a meet semilattice with 0 and $L^{\prime}=\{[x] \mid$ $x \in L\}$, where $[x]=\{z \in L \mid \operatorname{ann}(z)=\operatorname{ann}(x)\}$. Then $L^{\prime}$ is a meet semilattice under the partial order $[x] \leqslant[y]$ if and only if $\operatorname{ann}(y) \subseteq \operatorname{ann}(x)$.

Proof. Since $\operatorname{ann}(x), \operatorname{ann}(y) \subseteq \operatorname{ann}(x \wedge y)$, we have $[x \wedge y] \leqslant$ $[x]$, $[y]$. We claim that $[x] \wedge[y]=[x \wedge y]$. Let $[t]$ be another lower bound of $[x]$ and $[y]$. Then $\operatorname{ann}(x), \operatorname{ann}(y) \subseteq \operatorname{ann}(t)$.



Figure 2: The graph $G_{E}(L)$ is finite, whereas $G_{E}\left(L_{1}\right)$ is infinite.

Now, we claim that $\operatorname{ann}(x \wedge y) \subseteq \operatorname{ann}(t)$. Let $z \in \operatorname{ann}(x \wedge y)$. Then $z \wedge x \in \operatorname{ann}(y) \subseteq \operatorname{ann}(t)$. This gives $x \wedge z \wedge t=0$; that is, $z \wedge t \in \operatorname{ann}(x) \subseteq \operatorname{ann}(t)$. Hence $z \wedge t=0$; that is, $z \in \operatorname{ann}(t)$. This proves that $\operatorname{ann}(x \wedge y) \subseteq \operatorname{ann}(t)$. Thus $[t] \leqslant[x \wedge y]$ and this yields $[x] \wedge[y]=[x \wedge y]$. Clearly, if $L$ is a finite lattice, then $L^{\prime}$ is also a lattice.

Lemma 3. $L^{\prime}$ is an SSC meet semilattice.

Proof. Let $0<[x] \varsubsetneqq[y]$. Then $\operatorname{ann}(y) \varsubsetneqq \operatorname{ann}(x)$. Hence there exists $z \in \operatorname{ann}(x)$ such that $z \notin \operatorname{ann}(y)$. Thus $0 \neq z \wedge y=t$. But then $[0]<[t] \leqslant[y]$ and $[t] \wedge[x]=[0]$. Thus $L^{\prime}$ is an SSC meet semilattice.

Now, we introduce the graph $G_{E}(L)$ of equivalence classes of zero divisors of a meet semilattice $L$ with 0 , denoted by $G_{E}(L)$.

Definition 4. Let $L$ be a meet semilattice with 0 . We associate a simple undirected graph with $L$ whose vertices are the equivalence classes of elements in $Z_{\{0\}}(L)^{*}$ and with each pair of distinct classes $[x]$ and $[y]$ joined by an edge if and only if $[x] \wedge[y]=\{0\}=[0]$.

We illustrate the concept of the graph of equivalence classes of zero divisor with an example, see Figure 1.

Consider the following two infinite lattices $L$ and $L_{1}$.

We can see that the zero divisor graph of lattices $L, G_{\{0\}}(L)$ is infinite, but the graph of equivalence classes of zero divisors of the lattice $L$, namely, $G_{E}(L)$, is finite. Also for the lattices $L_{1}$, $G_{\{0\}}\left(L_{1}\right)$, and $G_{E}\left(L_{1}\right)$ both are infinite, see Figure 2.

Corollary 5. Let $L$ be a meet semilattice with 0 . Then there exists an SSC meet semilattice $L^{\prime}$ (as constructed in Lemma 2) such that $G_{E}(L)=G_{\{0\}}\left(L^{\prime}\right)$. 
Proof. From the construction of $L^{\prime}$, it is clear that $G_{E}(L)=$ $G_{\{0\}}\left(L^{\prime}\right)$.

Hence from Corollary 5, it is clear that the study of the graph of equivalence classes of zero divisors of $L$ is nothing, but the study of zero divisor graphs of SSC meet semilattices.

Remark 6. Recall that a prime semi-ideal $P$ of a meet semilattice $L$ is an associated prime if it is the annihilator of some elements of $L$; that is, $P=\operatorname{ann}(x)$ for some nonzero $x \in L$. The set of associated primes is denoted by $\operatorname{Ass}(L)$. If Clique $\left(G_{\{0\}}(L)\right)<\infty$, then $\operatorname{Ass}(L)$ is nonempty and finite. Further, any maximal element of the family of annihilator semi-ideals is prime; see Halaš and Jukl [4, Lemma 2.4, 2.6]. Note that every zero divisor is contained in an annihilator ideal and maximal annihilators are associated primes; we have $Z_{\{0\}}(L) \subseteq \bigcup_{P \in \operatorname{Ass}(L)} P$. Clearly, the reverse inclusion is trivial. Hence $Z_{\{0\}}(L)=\bigcup_{P \in \operatorname{Ass}(L)} P$; that is, the set of zerodivisors of $L$ equals the union of all associated primes of $L$. It is obvious to prove that a map $\phi: \operatorname{Ass}(L) \rightarrow V\left(G_{E}(L)\right)$ defined by $\phi(P)=[x]$, where $P=\operatorname{ann}(x)$ is an injective map. We adopt the conventions of Spiroff and Wickham [15], and by a slight abuse of terminology we will refer to the vertex $[y]$ as an associated prime if $\operatorname{ann}(y) \in \operatorname{Ass}(L)$. It will be clear from context whether $[y]$ refers to an equivalence class, a vertex, or a specific annihilator.

Theorem 7. Let $L$ be a meet semilattice with 0 , and let Clique $\left(G_{\{0\}}(L)\right)<\infty$. If ann $(x)$ and ann $(y)$ are distinct prime semi-ideals of $L$, then $[x]$ and $[y]$ are adjacent in $G_{E}(L)$. Furthermore, every vertex of $G_{E}(L)$ is either an associated prime semi-ideal or adjacent to a maximal semi-ideal in $\mathscr{L}=$ $\{\operatorname{ann}(x) \mid(0 \neq) x \in L\}$.

Proof. Since $\operatorname{ann}(x)$ and $\operatorname{ann}(y)$ are distinct, we have $x \wedge$ $y=0$. This further gives that $[x] \wedge[y]=[x \wedge y]=[0]$. Thus $[x]$ and $[y]$ are adjacent. Furthermore, suppose that $[x] \in V\left(G_{E}(L)\right)$ is not an associated prime semi-ideal. Then there exists $[z] \in V\left(G_{E}(L)\right)$ such that $[x] \wedge[z]=[0]=$ $\{0\}$. Hence $x \in \operatorname{ann}(z)$. We claim that $\operatorname{ann}(z)$ is maximal. Otherwise, since $\operatorname{Clique}\left(G_{\{0\}}(L)\right)<\infty, \mathscr{L}$ has a maximal element, say $\operatorname{ann}\left(z^{\prime}\right)\left(z^{\prime} \neq 0\right)$ containing $\operatorname{ann}(z)$, then $x \in$ $\operatorname{ann}(z) \subseteq \operatorname{ann}\left(z^{\prime}\right)$. It is clear that every maximal element of $\mathscr{L}$ is prime. Therefore $x \wedge z^{\prime}=0$. Thus there is an edge between $[x]$ and $\left[z^{\prime}\right]$. This completes the proof.

Remark 8. For a Boolean lattice, $[a]=\{a\}$ for every $a \in Z_{\{0\}}(L)$, and hence in this case, $G_{E}(L)$ and $G_{\{0\}}(L)$ are isomorphic. This fact is illustrated in Figure 3.

In view of Remark 8, we raise the following problem.

Question 1. Find a class of meet semilattices $\mathscr{L}$ such that $G_{\{0\}}(L) \cong G_{E}(L)$ for $L \in \mathscr{L}$.

Before we answer this question, we need the following result which follows from the definition of SSC meet semilattice.

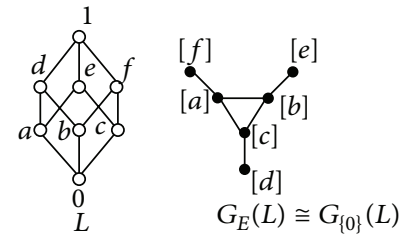

FIgURE 3: The isomorphism of $G_{E}(L)$ and $G_{\{0\}}(L)$.

Lemma 9. Let $L$ be a meet semilattice with 0 . Then $L$ is SSC if and only if for $a, b \in L, \operatorname{ann}(a)=a n n(b)$ implies $a=b$.

Proof. Suppose that $L$ is SSC. Further, assume that there exist $a, b \in L$ such that $\operatorname{ann}(a)=\operatorname{ann}(b)$ and $a \neq b$. Since $L$ is SSC, there exist $c \in L$ such that $0<c \leqslant a$ and $c \wedge b=0$. This gives $c \in \operatorname{ann}(b)=\operatorname{ann}(a)$, a contradiction to the fact that $c \neq 0$. Hence $a=b$.

Theorem 10. Let $L$ be a finite semicomplemented meet semilattice. Then $\phi: G_{\{0\}}(L) \rightarrow G_{E}(L)$ is a graph isomorphism if and only if $L$ is SSC.

Proof. Let $L$ be a SSC meet semilattice. Then $G_{E}(L) \cong G_{\{0\}}(L)$ follows from Lemma 9.

Conversely, assume that $G_{E}(L) \cong G_{\{0\}}(L)$. First, we prove that $[a]=\{a\}, \forall a \in Z_{\{0\}}(L)^{*}$. Let $b \in[a]$ for $a \neq b \in$ $Z_{\{0\}}(L)^{*}$. Then we have $[a]=[b]$ for $a \neq b$, a contradiction to $\left|V\left(G_{E}(L)\right)\right|=\left|V\left(G_{\{0\}}(L)\right)\right|$. Now, let $a<b(a \neq 0)$. Since $L$ is semicomplemented, $a, b \in Z_{\{0\}}(L)^{*}$. This gives $\operatorname{ann}(b) \varsubsetneqq$ $\operatorname{ann}(a)$. Otherwise, if $\operatorname{ann}(a)=\operatorname{ann}(b)$, then $b \in[a]=\{a\}$, a contradiction. From the proof of Lemma 9, it is clear that $L$ is SSC.

If we consider that the $\phi: G_{\{0\}}(L) \rightarrow G_{E}(L)$ is a graph isomorphism defined by $\phi(a)=[a]$, then we do not need even the finiteness of $L$.

Theorem 11. Let $L$ be a semicomplemented meet semilattice. Then $\phi: G_{\{0\}}(L) \rightarrow G_{E}(L)$ is a graph isomorphism defined by $\phi(a)=[a]$ if and only if $L$ is SSC.

Proof. Let $L$ be a SSC meet semilattice. Then $G_{E}(L) \cong G_{\{0\}}(L)$ follows from Lemma 9.

Conversely, assume that $\phi: G_{\{0\}}(L) \rightarrow G_{E}(L)$ is a graph isomorphism defined by $\phi(a)=[a]$. This gives $[a]=$ $\{a\}$, for all $a \in Z_{\{0\}}(L)^{*}$. Let $a<b(a \neq 0)$. Since $L$ is semicomplemented, $a, b \in Z_{\{0\}}(L)^{*}$. This gives ann $(b) \varsubsetneqq$ $\operatorname{ann}(a)$. From the proof of Lemma 9, it is clear that $L$ is SSC.

From Figure 4, it is clear that for the posets $P_{1}, P_{2}$, $G_{\{0\}}\left(P_{1}\right) \cong G_{\{0\}}\left(P_{2}\right) \nRightarrow P_{1} \cong P_{2}$. Hence, it is worth to study the following problem.

Problem 12. Find a class of posets $\mathscr{P}$ such that $G_{\{0\}}\left(P_{1}\right) \cong$ $G_{\{0\}}\left(P_{2}\right)$ if and only if $P_{1} \cong P_{2}$ for the posets $P_{1}, P_{2} \in \mathscr{P}$. 

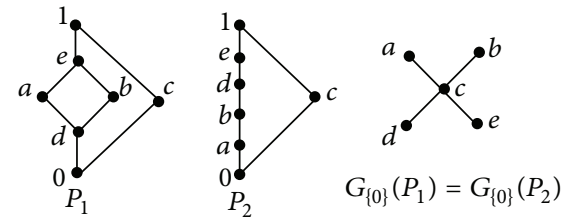

FIGURE 4: Nonisomorphic lattices with isomorphic zero divisor graphs.

In [8], Joshi and Khiste answered this problem in the case of Boolean posets. More details about Boolean posets can be found in Waphare and Joshi [21].

Theorem 13 (Joshi and Khiste [8, Theorem 2.11]). Let $P_{1}$ and $P_{2}$ be Boolean posets. Then $G_{\{0\}}\left(P_{1}\right) \cong G_{\{0\}}\left(P_{2}\right)$ if and only if $P_{1} \cong P_{2}$.

It is well known that there is a $1-1$ correspondence between Boolean algebras and Boolean rings.

A consequence of the above theorem is the following result.

Corollary 14 (LaGrange [16, Theorem 4.1], Mohammadian [22, Theorem 7]). Let $P_{1}$ and $P_{2}$ be Boolean algebras. Then $G_{\{0\}}\left(P_{1}\right) \cong G_{\{0\}}\left(P_{2}\right)$ if and only if $P_{1} \cong P_{2}$.

Now, we are ready to extend this result to the class of SSC meet semilattices. Note that every Boolean lattice is SSC but not conversely. Consider the lattice $L_{1}$ depicted in Figure 2 is SSC but not a Boolean lattice.

Theorem 15. Let $L_{1}$ and $L_{2}$ be bounded SSC meet semilattices. Then the following statements are equivalent:

(1) $G_{E}\left(L_{1}\right) \cong G_{E}\left(L_{2}\right)$;

(2) $G_{\{0\}}\left(L_{1}\right) \cong G_{\{0\}}\left(L_{2}\right)$;

(3) $L_{1} \cong L_{2}$.

Proof. The equivalence of statements (1) and (2) follows from Lemma 9.

$(2) \Rightarrow$ (3) : Since $L_{1}$ and $L_{2}$ are semicomplemented, $V\left(G_{\{0\}}\left(L_{i}\right)\right)=L_{i} \backslash\left\{0_{L_{i}}, 1_{L_{i}}\right\}$ for $i=1,2$. Let $\phi: V\left(G_{\{0\}}\left(L_{1}\right)\right) \rightarrow$ $V\left(G_{\{0\}}\left(L_{2}\right)\right)$ be a graph isomorphism. Define a map $f: L_{1} \rightarrow$ $L_{2}$ such that $f(a)=\phi(a)$, for all $a \in V\left(G_{\{0\}}\left(L_{1}\right)\right)$ along with $f\left(0_{L_{1}}\right)=0_{L_{2}}$ and $f\left(1_{L_{1}}\right)=1_{L_{2}}$, where $0_{L_{i}}\left(1_{L_{i}}\right)$ is the smallest (largest) element of $L_{i}$. Since $\phi$ is bijective, $f$ is bijective. We claim that $f$ is a biorder preserving map.

Let $a \leqslant b$. First, we show that $f(a) \leqslant f(b)$. If any of $a, b$ is either $0_{L_{1}}$ or $1_{L_{1}}$, then we are through. Hence assume that $a, b \notin\left\{0_{L_{1}}, 1_{L_{1}}\right\}$. Suppose that $f(a) \nless f(b)$; that is, $\phi(a) \nless \phi(b)$. Since $L_{2}$ is SSC, there exists $\phi(c) \in L_{2}$ such that $0_{L_{2}}<\phi(c) \leqslant \phi(a)$ and $\phi(c) \wedge \phi(b)=0_{L_{2}}$. Since $b \neq 0_{L_{1}}$, we have $\phi(b) \neq 0_{L_{2}}$. This gives that $\phi(c)$ and $\phi(b)$ are adjacent in $G_{\{0\}}\left(L_{2}\right)$. Since $\phi$ is a graph isomorphism, we have that $c$ and $b$ are adjacent which further imply that $c \wedge b=0_{L_{1}}$. Hence, $c \wedge a=0_{L_{1}}$ in $L_{1}$. Thus $a$ and $c$ are adjacent in $G_{\{0\}}\left(L_{1}\right)$. Hence, we have $\phi(c) \wedge \phi(a)=0_{L_{2}}$. But $\phi(c) \leqslant \phi(a)$ gives $\phi(c)=0_{L_{2}}$, a contradiction. Hence $f(a) \leqslant f(b)$ in $L_{2}$.
Conversely, assume that $f(a) \leqslant f(b)$ in $L_{2}$. We claim that $a \leqslant b$. If any of $f(a)$ or $f(b)$ is $0_{L_{2}}$ or $1_{L_{2}}$, then we are through. Let us assume that $f(a), f(b) \in L_{2} \backslash\left\{0_{L_{2}}, 1_{L_{2}}\right\}$. This yields $a, b \notin\left\{0_{L_{1}}, 1_{L_{1}}\right\}$. Now, suppose on the contrary that $a \nless b$ for $a, b \notin\left\{0_{L_{1}}, 1_{L_{1}}\right\}$. Since $L_{1}$ is SSC, there exists $c \in L_{1}$ such that $0_{L_{1}}<c \leqslant a$ and $c \wedge b=0_{L_{1}}$. Thus $c$ and $b$ are adjacent in $G_{\{0\}}\left(L_{1}\right)$. Then $\phi(c)$ and $\phi(b)$ are adjacent in $G_{\{0\}}\left(L_{2}\right)$. Then $\phi(c) \wedge \phi(b)=0_{L_{2}}$ in $L_{2}$. Replacing the role of $a, b$ in the above proof by $c, a$, respectively, we have $\phi(c) \leqslant \phi(a)$. This implies that $\phi(c) \leqslant \phi(a) \leqslant \phi(b)$. But this gives $\phi(c)=\phi(c) \wedge \phi(b)=$ $0_{L_{2}}$, a contradiction. Hence $a \leqslant b$. Thus $L_{1} \cong L_{2}$.

$$
\text { (3) } \Rightarrow(2) \text { : Obvious. }
$$

Lemma 16. Let $L_{1}$ be a bounded SSC meet semilattice, and $L_{2}$ be a bounded semicomplemented meet semilattice such that $G_{\{0\}}\left(L_{1}\right) \cong G_{\{0\}}\left(L_{2}\right)$. Then $L_{2}$ is also SSC.

Proof. Since $L_{1}$ and $L_{2}$ are semicomplemented, $V\left(G_{\{0\}}\left(L_{i}\right)\right)=$ $L_{i} \backslash\left\{0_{L_{i}}, 1_{L_{i}}\right\}$ for $i=1,2$. Let $\phi: V\left(G_{\{0\}}\left(L_{1}\right)\right) \rightarrow V\left(G_{\{0\}}\left(L_{2}\right)\right)$ be a graph isomorphism. Define a map $f: L_{1} \rightarrow L_{2}$ such that $f(a)=\phi(a)$, for all $a \in V\left(G_{\{0\}}\left(L_{1}\right)\right)$ along with $f\left(0_{L_{1}}\right)=0_{L_{2}}$ and $f\left(1_{L_{1}}\right)=1_{L_{2}}$, where $0_{L_{i}}\left(1_{L_{i}}\right)$ is the smallest (largest) element of $L_{i}$. Since $\phi$ is a bijective map, $f$ is bijective. Let $f(a)<f(b)$ in $L_{2}$. Since $L_{2}$ is semicomplemented, and if $f(a)=0_{L_{2}}$ or $f(b)=1_{L_{2}}$, then we are through. Hence assume that $f(a) \neq 0_{L_{2}}$ and $f(b) \neq 1_{L_{2}}$. Then clearly, $a \neq b$ and $b \neq 1_{L_{1}}$. Since $L_{1}$ is SSC and $a \wedge b<b$, there exists $0_{L_{1}}<c \leqslant b$ and $c \wedge a=0_{L_{1}}$. Since $c$ and $a$ are adjacent in $G_{\{0\}}\left(L_{1}\right)$, we have $\phi(c)$ and $\phi(a)$ are adjacent in $G_{\{0\}}\left(L_{2}\right)$; that is, $\phi(c) \wedge \phi(a)=0_{L_{2}}$. Further $b$ and $c$ are nonadjacent in $G_{\{0\}}\left(L_{1}\right)$. Then $\phi(b)$ and $\phi(c)$ are nonadjacent in $G_{\{0\}}\left(L_{2}\right)$. Consider $0_{L_{2}} \neq \phi(b) \wedge \phi(c)=$ $f(b) \wedge f(c)=f(d)$ say. Clearly, $0_{L_{2}}<f(d) \leqslant f(b)$ and $f(d) \wedge f(a)=0_{L_{2}}\left(\right.$ as $\phi(c) \wedge \phi(a)=0_{L_{2}}$ implies $f(c) \wedge f(a)=$ $0_{L_{2}}$ ). Thus $L_{2}$ is SSC.

Theorem 17. Let $L_{1}$ and $L_{2}$ be bounded semicomplemented meet semilattices. If $G_{E}\left(L_{1}\right) \cong G_{\{0\}}\left(L_{2}\right)$, then $L_{2}$ is SSC.

Proof. For the given lattice $L_{1}$, we can construct an SSC meet semilattice $L_{1}^{\prime}$ (as given in Lemma 2), such that $G_{E}\left(L_{1}\right) \cong$ $G_{\{0\}}\left(L_{1}^{\prime}\right)$. This gives $G_{\{0\}}\left(L_{1}^{\prime}\right) \cong G_{\{0\}}\left(L_{2}\right)$. By Lemma 16, $L_{2}$ is SSC.

The above result is analogues to the following result of Anderson and LaGrange [14].

Theorem 18 (Anderson and LaGrange [14, Theorem 2.6]). Let $R$ and $S$ be commutative reduced rings with $1 \neq 0$ and $Z(S) \neq\{0\}$. If $\Gamma_{E}(R) \cong \Gamma(S)$, then $S$ is a Boolean ring.

We quote the result of Joshi [6, Theorem 2.4] when the corresponding poset is a meet semilattice.

Theorem 19 (Joshi [6, Theorem 2.4]). Let L be a meet semilattice with 0 . Then the graph $G_{\{0\}}(L)$ is connected and $\operatorname{diam}\left(G_{\{0\}}(L)\right) \leqslant 3$.

Theorem 20. Let $L$ be a meet semilattice with 0 . Then the graph $G_{E}(L)$ is connected and $\operatorname{diam}\left(G_{E}(L)\right) \leqslant$ $\operatorname{diam}\left(G_{\{0\}}(L)\right) \leqslant 3$. 
Proof. Follows from Corollary 5, Theorem 19, and the fact that adjacency in $G_{E}(L)$ implies adjacency in $G_{\{0\}}(L)$. Hence $\operatorname{diam}\left(G_{E}(L)\right) \leqslant \operatorname{diam}\left(G_{\{0\}}(L)\right) \leqslant 3$.

Theorem 21. Let $L$ be an SSC lattice. Then $G_{\{0\}}(L)$ is either $K_{2}$ or contains a cycle of length 3 . Hence girth $\left(G_{\{0\}}(L)\right)=3$.

Proof. If $V\left(G_{\{0\}}(L)\right)=2$, then $G_{\{0\}}(L) \cong K_{2}$, by Theorem 19 . Now assume that $\left|V\left(G_{\{0\}}(L)\right)\right| \geqslant 3$. Choose $a, b, c \in$ $V\left(G_{\{0\}}(L)\right)$ such that $a-b-c$ is a path with $a \neq c$ and $a \wedge c \neq 0$. Since $L$ is SSC and without loss of generality assume that $a \wedge c<a$, there exists $x$ such that $0<x \leqslant a$ and $c \wedge x=0$. Clearly, $x \in V\left(G_{\{0\}}(L)\right)$. Since $a \wedge b=0$, we have $x \wedge b=0$. Thus we have a cycle $b-x-c-b$ of length 3 .

An immediate consequence of the above theorem is the following corollary.

Corollary 22. Let $L$ be a meet semilattice with 0 ; then $G_{E}(L)$ is either $K_{2}$ or contains a cycle of length 3 . Hence, if $G_{E}(L) \not K_{2}$, then $\operatorname{girth}\left(G_{E}(L)\right)=3=\operatorname{girth}\left(G_{\{0\}}(L)\right)$.

Proof. It follows from Corollary 5 and Theorem 21.

Remark 23. In the above result, the condition of existence of a cycle in $G_{E}(L)$ is necessary. In Figure 1, we can observe that $G_{\{0\}}(L)$ has a cycle but $G_{E}(L)$ has no cycle.

\section{Correlation between Diameter of $G_{\{0\}}(L)$ and $G_{E}(L)$}

The following result gives the correlation between diameter of $G_{\{0\}}(L)$ and $G_{E}(L)$.

Theorem 24. The following statements are true for a meet semilattice $L$ with 0 .

(a) If $\operatorname{diam}\left(G_{\{0\}}(L)\right)=1$, then $\operatorname{diam}\left(G_{E}(L)\right)=1$.

(b) If $\operatorname{diam}\left(G_{\{0\}}(L)\right)=2$, then $\operatorname{diam}\left(G_{E}(L)\right)=1$ or 2 .

(c) If $\operatorname{diam}\left(G_{\{0\}}(L)\right)=3$, if and only if $\operatorname{diam}\left(G_{E}(L)\right)=3$.

(d) If $\operatorname{diam}\left(G_{E}(L)\right)=1$, then $\operatorname{diam}\left(G_{\{0\}}(L)\right)=1$ or 2 .

(e) If $\operatorname{diam}\left(G_{E}(L)\right)=2$, then $\operatorname{diam}\left(G_{\{0\}}(L)\right)=2$.

Proof. (a) It is clear that $\operatorname{diam}\left(G_{E}(L)\right) \neq 0$. Hence the result follows from Theorem 20.

(b) Suppose that $\operatorname{diam}\left(G_{\{0\}}(L)\right)=2$. So we have a path $a-b-c$ in $G_{\{0\}}(L)$. We have two possibilities.

Case 1. If $[a],[b]$, and $[c]$ are distinct vertices of $G_{E}(L)$, we get the path $[a]-[b]-[c]$. Hence by Theorem $20, \operatorname{diam}\left(G_{E}(L)\right)=$ 2 .

Case 2. The only possibility in this case is $[a]=[c]$, and hence, $\operatorname{diam}\left(G_{E}(L)\right)=1$.

(c) Let $\operatorname{diam}\left(G_{\{0\}}(L)\right)=3$. Then there exists a path $a-c-$ $d-b$. Since $c \in \operatorname{ann}(a)$ gives $[a] \neq[b]$; otherwise $d(a, b)=2$, a contradiction. Now assume that $d([a],[b])=2$ in $G_{E}(L)$. Then there exists $[x] \in V\left(G_{E}(L)\right)$ such that $[a]-[x]-[b]$. This yields that $d(a, b)=2$, again a contradiction. Thus by



FIgURE 5: $\operatorname{diam}\left(G_{E}(L)\right)=\operatorname{diam}\left(G_{\{0\}}(L)\right)=1$.



Figure 6: $\operatorname{diam}\left(G_{E}(L)\right)=\operatorname{diam}\left(G_{\{0\}}(L)\right)=2$.

Theorem 20, $\operatorname{diam}\left(G_{E}(L)\right)=3$. The converse follows from Theorem 20.

(d) Suppose that $\operatorname{diam}\left(G_{E}(L)\right)=1$. Then $\operatorname{diam}\left(G_{\{0\}}(L)\right) \neq 3$, by $(c)$. Hence the result.

(e) Suppose that $\operatorname{diam}\left(G_{E}(L)\right)=2$; then there exists a path $[a]-[c]-[b]$ in $G_{E}(L)$. This yields a path $a-c-b$ in $G_{\{0\}}(L)$. Thus $\operatorname{diam}\left(G_{\{0\}}(L)\right) \neq 1$. The result follows from $(c)$ and $\operatorname{diam}\left(G_{\{0\}}(L)\right) \neq 3$.

Remark 25. The assertions $(a)$ and $(d)$ of Theorem 24 are justified in Figures 1 and 5, whereas the assertions $(b)$ and $(e)$ are justified in Figures 5 and 6. Figure 3 verifies the assertion (c).

Corollary 26. Let $L$ be a meet semilattice with 0 . Then $\operatorname{diam}\left(G_{E}(L)\right) \leqslant 2$ if and only if $\operatorname{diam}\left(G_{\{0\}}(L)\right) \leqslant 2$.

\section{Cut Vertices in $G_{E}(L)$}

In this section, we examine the properties of cut vertices of $G_{E}(L)$.

Definition 27. A lattice $L$ with 0 is said to be 0 -distributive, if $a \wedge b=a \wedge c=0$ implies that $a \wedge(b \vee c)=0$; Varlet [23]. More details about 0 -distributive posets can be found in Joshi and Waphare [24]; see also Joshi and Mundlik [25].

Theorem 28. Let $L$ be a 0 -distributive lattice. If $[a]$ is a cut vertex of $G_{E}(L)$, then $[a] \cup\{0\}$ forms an ideal of $L$.

Proof. Let $[a]$ be a cut vertex of $G_{E}(L)$. Let $a_{1}$ and $a_{2}$ be nonzero elements of $[a]$. Since $a_{1}, a_{2} \in[a]$, ann $\left(a_{1}\right)=$ $\operatorname{ann}(a)=\operatorname{ann}\left(a_{2}\right)$. Now, it is enough to show that $\operatorname{ann}\left(a_{1} \vee\right.$ $\left.a_{2}\right)=\operatorname{ann}(a)$. Clearly, ann $\left(a_{1} \vee a_{2}\right) \subseteq \operatorname{ann}(a)$.

Let $t \in \operatorname{ann}(a)$. This gives $t \wedge a_{1}=t \wedge a_{2}=t \wedge a=0$. Since $L$ is 0 -distributive, we have $t \wedge\left(a_{1} \vee a_{2}\right)=0$; that is, $t \in \operatorname{ann}\left(a_{1} \vee a_{2}\right)$. Thus ann $(a) \subseteq \operatorname{ann}\left(a_{1} \vee a_{2}\right)$. This implies that $a_{1} \vee a_{2} \in[a]$.

Now suppose that $x \in[a] \cup\{0\}$ and $y \leqslant x$, to show that $y \in$ $[a] \cup\{0\}$. If $x=0$ or $y=0$, then $y \in[a] \cup\{0\}$. Let $y \neq 0$ and $y \leqslant$ $x$. Then $\operatorname{ann}(x) \subseteq \operatorname{ann}(y)$. Since $[a]$ is a cut vertex of $G_{E}(L)$, 
therefore for any two arbitrary vertices $[b],[c] \in V\left(G_{E}(L)\right)$, we have a path $[b]-[a]-[c]$. Therefore $b \in \operatorname{ann}(a)=\operatorname{ann}(x)$; that is, $b \wedge x=0$, which further yields $b \wedge y=0$. Similarly, $c \wedge y=0$. Therefore, we get a path $[b]-[y]-[c]$. Since $[a]$ is cut vertex, $[a]=[y]$. Thus $[a] \cup\{0\}$ is an ideal.

Remark 29. It is clear from the proof of the above theorem that in a general lattice, if $[a]$ is a cut vertex of $G_{E}(L)$, then $[a] \cup\{0\}$ is a semi-ideal.

Lemma 30. Let $L$ be a meet semilattice with 0 . If $[a]$ is a cut vertex of $G_{E}(L)$, then ann $(a)$ is maximal in $\mathscr{L}=\{\operatorname{ann}(x) \mid$ $0 \neq x \in L\}$. Hence ann $(a)$ is a prime semi-ideal.

Proof. Let $[a]$ be a cut vertex of $G_{E}(L)$, and let $G_{1}$ and $G_{2}$ be mutually separated subgraphs of $G_{E}(L)$ with $V\left(G_{1} \cup G_{2}\right)=$ $V\left(G_{E}(L)\right) \backslash[a]$. Let $[b] \in G_{1}$ and $[c] \in G_{2}$, therefore we have the path $[b]-[a]-[c]$. Suppose that ann $(a) \subseteq \operatorname{ann}(x)$ for some $\operatorname{ann}(x) \in \mathscr{L}$. Since $[b]-[a]$ is an edge, $b \in \operatorname{ann}(a) \subseteq \operatorname{ann}(x)$. Then $[b] \wedge[x]=\{0\}$. Similarly, $[c] \wedge[x]=\{0\}$. Thus we have another path $[b]-[x]-[c]$ passing through of $[x]$. Since $[a]$ is a cut vertex of $G_{E}(L)$, then $[x]=[a]$. Thus $\operatorname{ann}(x)=\operatorname{ann}(a)$. It is easy to prove that $\operatorname{ann}(a)$ is a prime semi-ideal.

Lemma 31. If $[a]$ is a cut vertex of $G_{E}(L)$, then all other associated primes of $G_{E}(L)$ are contained in only one component of $G_{E}(L) \backslash[a]$.

Proof. Suppose that $G_{1}$ and $G_{2}$ are two mutually separated connected components of $G_{E}(L) \backslash[a]$, and each contains an associated prime. It is easy to observe that the associated primes are adjacent, and hence $G_{1}, G_{2}$ are connected, a contradiction.

Alizadeh et al. [2] raised the following problem.

Problem 32. Characterize those posets $P$ for which $\left(\operatorname{diam}\left(G_{\{0\}}(P)\right), \operatorname{girth}\left(G_{\{0\}}(P)\right)\right)=(2,3)$ or $(3,3)$.

In view of Corollary 5 and the following result, it is clear that for an SSC meet semilattice $L$, $\left(\operatorname{diam}\left(G_{\{0\}}(L)\right), \operatorname{girth}\left(G_{\{0\}}(L)\right)\right)=(3,3)$.

Theorem 33. Let $L$ be a meet semilattice with 0 . If $\left|V\left(G_{E}(L)\right)\right| \geqslant 4$ and $G_{E}(L)$ have at least 2 cut vertices, then $\left(\operatorname{diam}\left(G_{E}(L)\right), \operatorname{girth}\left(G_{E}(L)\right)=(3,3)\right.$.

Proof. Let $[a]$ and $[b]$ be cut vertices of $G_{E}(L)$. Since $[a]$ is a cut vertex of $G_{E}(L)$, there is some $\left[a_{1}\right]$ such that any path connecting $\left[a_{1}\right]$ and $[b]$ must include $[a]$. Similarly, since $[b]$ is a cut vertex, there is some $\left[b_{1}\right]$ such that any path connecting $\left[b_{1}\right]$ and $[a]$ must include $[b]$. Clearly, $\left[a_{1}\right] \neq\left[b_{1}\right]$ and any path from $\left[a_{1}\right]$ to $\left[b_{1}\right]$ must include $[a]$ and $[b]$ and so $\operatorname{diam}([a],[b]) \geqslant 3$. By Theorem $20, \operatorname{diam}\left(G_{E}(L)\right)=3$. Thus $\left(\operatorname{diam}\left(G_{E}(L)\right), \operatorname{girth}\left(G_{E}(L)\right)=(3,3)\right.$, by Corollary 22 .

It is easy to see that Figure 3 fulfills the conditions of Theorem 33.

\section{Beck's Conjecture for $G_{E}(L)$}

Joshi [6] (see also Halaš and Jukl [4], Nimbhorkar et al. [26], and $\mathrm{Lu}$ and $\mathrm{Wu}[12]$ ) proved that Beck's Conjecture is true for the zero divisor graphs of posets. We quote this result when the poset is a meet semilattice and ideal is a zero ideal.

Theorem 34 (Joshi [6, Theorem 2.9]). Let $L$ be a meet semilattice with 0. IfClique $\left(G_{\{0\}}(L)\right)<\infty$, then $\operatorname{Clique}\left(G_{\{0\}}(L)\right)=$ $\chi\left(G_{\{0\}}(L)\right)=n$, where $n$ is the number of minimal prime semiideals of $L$.

From Theorem 34 and Corollary 5, it is clear that Beck's Conjecture is true for the graph $G_{E}(L)$. In the sequel, we calculate the chromatic number of $G_{E}(L)$. As a preparation, we need the following easy lemma.

Lemma 35. Let $P$ be a minimal prime semi-ideal of a meet semilattice $L$ with 0 . Then for any $x \in P$, there exists $y \notin P$ such that $x \wedge y=0$.

Proof. It follows from the fact that $L \backslash P$ is a maximal filter of $L$ and $x \notin L \backslash P$.

Lemma 36. Let $L$ be a meet semilattice with 0 , and let $L^{\prime}$ be a meet semilattice with 0 (as constructed in Lemma 2). If $P$ is a minimal prime semi-ideal of $L$, then $P^{\prime}=\{[x] \mid x \in P\}$ is a minimal prime semi-ideal of $L^{\prime}$.

Proof. First, we prove that $P^{\prime}$ is a semi-ideal. Let $[y]<[x] \epsilon$ $P^{\prime}$. On the contrary, assume that $[y] \notin P^{\prime}$. Hence $\operatorname{ann}(y) \subseteq$ $P$. Further, $[y]<[x]$ gives $\operatorname{ann}(x) \varsubsetneqq \operatorname{ann}(y)$ which yields $\operatorname{ann}(x) \subseteq P$. Since $x \in P$, by Lemma 35 , there exists $y \notin P$ such that $x \wedge y=0$; that is, $y \in \operatorname{ann}(x)$. But then $y \in \operatorname{ann}(x) \subseteq$ $P$, a contradiction. Thus $P^{\prime}$ is a semi-ideal.

Let $[x] \wedge[y] \in P^{\prime}$. Then $x \wedge y \in P$. By primeness of $P$, either $x \in P$ or $y \in P$. This gives either $[x] \in P^{\prime}$ or $[y] \in P^{\prime}$. Thus $P^{\prime}$ is prime. Let $Q^{\prime}$ be a prime semi-ideal of $L^{\prime}$ such that $Q^{\prime} \varsubsetneqq P^{\prime}$. Hence there exists $[x] \in P^{\prime}$ such that $[x] \notin Q^{\prime}$. Again by Lemma 35, there exists $y \notin P$ such that $x \wedge y=0$. This gives $[x] \wedge[y]=[0] \in Q^{\prime}$. But then $[y] \in Q^{\prime} \subseteq P^{\prime}$, a contradiction. Thus $P^{\prime}$ is a minimal prime semi-ideal of $L^{\prime}$.

On similar lines, we can prove the following result.

Lemma 37. Let $L$ be a meet semilattice with 0 , and let $L^{\prime}$ be a meet semilattice with 0 (as constructed in Lemma 2). If $P^{\prime}$ is a minimal prime semi-ideal of $L^{\prime}$, then $P=\left\{x \mid[x] \in P^{\prime}\right\}$ is a minimal prime semi-ideal of $L$.

Let us denote the set of all minimal prime semi-ideals of $L$ by $\operatorname{Min}_{s}(L)$.

Theorem 38. Let $L$ be a meet semilattice with 0 , and let $L^{\prime}$ be a meet semilattice with 0 (as constructed in Lemma 2). Let $\phi: \operatorname{Min}_{s}(L) \rightarrow \operatorname{Min}_{s}\left(L^{\prime}\right)$ be a map defined by $\phi(P)=P^{\prime}=$ $\{[x] \mid x \in P\}$. Then $\phi$ is bijective.

Proof. Let $\phi: \operatorname{Min}_{s}(L) \rightarrow \operatorname{Min}_{s}\left(L^{\prime}\right)$ be a map defined by $\phi(P)=P^{\prime}=\{[x] \mid x \in P\}$. By Lemma 36, $P^{\prime}$ is a minimal prime semi-ideal. First, we show that $\phi$ is one-to-one. Let 
$\phi(P)=\phi(Q)$. Then for $x \in P$, we have $[x] \in P^{\prime}=\phi(P)=$ $\phi(Q)=Q^{\prime}$. This gives $x \in Q$. Hence $P \subseteq Q$. Similarly, $Q \subseteq P$. Thus $\phi$ is one-to-one. Let $Q^{\prime}$ be any minimal prime semi-ideal in $L^{\prime}$. Then $Q=\left\{x \mid[x] \in Q^{\prime}\right\}$ is a minimal prime semiideal of $L$, by Lemma 37. It is clear that $\phi(Q)=Q^{\prime}$. Thus $\phi$ is onto.

With this preparation, we now prove Beck's Conjecture for $G_{E}(L)$.

Theorem 39. Let $L$ be a meet semilattice with 0 . If Clique $\left(G_{E}(L)\right)<\infty$, then $\chi\left(G_{E}(L)\right)=\chi\left(G_{\{0\}}(L)\right)=$ Clique $\left(G_{E}(L)\right)=\operatorname{Clique}\left(G_{\{0\}}(L)\right)=n$, where $n$ is the number of all minimal prime semi-ideals of $L$.

Proof. Let Clique $\left(G_{E}(L)\right)<\infty$. Then Clique $\left(G_{\{0\}}\left(L^{\prime}\right)\right)<\infty$, by Corollary 5 , where $L^{\prime}$ is the semilattice constructed as in Lemma 2. By Theorem 34, Clique $\left(G_{\{0\}}\left(L^{\prime}\right)\right)=\chi\left(G_{\{0\}}\left(L^{\prime}\right)\right)=$ $n$, where $n$ is the number of minimal prime semi-ideals of $L^{\prime}$. Now, by Theorem 38, the number of minimal prime semi-ideals of $L$ is also $n$. Thus we have Clique $\left(G_{E}(L)\right)=$ $\chi\left(G_{E}(L)\right)=n$.

\section{Conflict of Interests}

The authors declare that there is no conflict of interests regarding the publication of this paper.

\section{References}

[1] I. Beck, "Coloring of commutative rings," Journal of Algebra, vol. 116, no. 1, pp. 208-226, 1988.

[2] M. Alizadeh, A. K. Das, H. R. Maimani, M. R. Pournaki, and S. Yassemi, "On the diameter and girth of zero-divisor graphs of posets," Discrete Applied Mathematics, vol. 160, no. 9, pp. 13191324, 2012.

[3] M. Alizadeh, H. R. Maimani, M. R. Pournaki, and S. Yassemi, "An ideal theoretic approach to complete partite zero-divisor graphs of posets," Journal of Algebra and its Applications, vol. 12, no. 2, Article ID 1250148, 11 pages, 2013.

[4] R. Halaš and M. Jukl, "On Beck's coloring of posets," Discrete Mathematics, vol. 309, no. 13, pp. 4584-4589, 2009.

[5] R. Halaš and H. Länger, "The zero divisor graph of a qoset," Order, vol. 27, no. 3, pp. 343-351, 2010.

[6] V. Joshi, "Zero divisor graph of a poset with respect to an ideal," Order, vol. 29, no. 3, pp. 499-506, 2012.

[7] V. Joshi and A. Khiste, "On the zero divisor graphs of pmlattices," Discrete Mathematics, vol. 312, no. 12-13, pp. 20762082, 2012.

[8] V. Joshi and A. Khiste, "On the zero divisor graph of a Boolean poset," Mathematica Slovaca. In press.

[9] V. Joshi and A. Khiste, "Complement of the zero divisor graph of a lattice," Bulletin of the Australian Mathematical Society, vol. 89, pp. 177-190, 2014.

[10] V. Joshi, B. N. Waphare, and H. Y. Pourali, "Zero divisor graphs of lattices and primal ideals," Asian-European Journal of Mathematics, vol. 5, no. 3, Article ID 1250037, 9 pages, 2012.

[11] V. Joshi, B. N. Waphare, and H. Y. Pourali, "On generalized zero divisor graph of a poset," Discrete Applied Mathematics, vol. 161, no. 10-11, pp. 1490-1495, 2013.
[12] D. Lu and T. Wu, "The zero-divisor graphs of posets and an application to semigroups," Graphs and Combinatorics, vol. 26, no. 6, pp. 793-804, 2010.

[13] B. Allen, E. Martin, E. New, and D. Skabelund, "Diameter, girth and cut vertices of the graph of equivalence classes of zerodivisors," Involve, vol. 5, no. 1, pp. 51-60, 2012.

[14] D. F. Anderson and J. D. LaGrange, "Commutative Boolean monoids, reduced rings, and the compressed zero-divisor graph," Journal of Pure and Applied Algebra, vol. 216, no. 7, pp. 1626-1636, 2012.

[15] S. Spiroff and C. Wickham, "A zero divisor graph determined by equivalence classes of zero divisors," Communications in Algebra, vol. 39, no. 7, pp. 2338-2348, 2011.

[16] J. D. LaGrange, "Complemented zero-divisor graphs and Boolean rings," Journal of Algebra, vol. 315, no. 2, pp. 600-611, 2007.

[17] M. F. Janowitz, “Section semicomplemented lattices," Mathematische Zeitschrift, vol. 108, pp. 63-76, 1968.

[18] N. K. Thakare, S. Maeda, and B. N. Waphare, "Modular pairs, covering property and related results in posets," The Journal of the Indian Mathematical Society, vol. 70, no. 1-4, pp. 229-253, 2003.

[19] N. K. Thakare, M. M. Pawar, and B. N. Waphare, "Modular pairs, standard elements, neutral elements and related results in partially ordered sets," The Journal of the Indian Mathematical Society, vol. 71, no. 1-4, pp. 13-53, 2004.

[20] V. Joshi, "On completion of section semicomplemented posets," Southeast Asian Bulletin of Mathematics, vol. 31, no. 5, pp. 881892, 2007.

[21] B. N. Waphare and V. V. Joshi, "On uniquely complemented posets," Order, vol. 22, no. 1, pp. 11-20, 2005.

[22] A. Mohammadian, "On zero-divisor graphs of Boolean rings," Pacific Journal of Mathematics, vol. 251, no. 2, pp. 375-383, 2011.

[23] J. C. Varlet, "A generalization of the notion of pseudocomplementedness," Bulletin de la Société Royale des Sciences de Liège, vol. 37, pp. 149-158, 1968.

[24] V. V. Joshi and B. N. Waphare, "Characterizations of 0distributive posets," Mathematica Bohemica, vol. 130, no. 1, pp. 73-80, 2005.

[25] V. Joshi and N. Mundlik, "Prime ideals in 0-distributive posets," Central European Journal of Mathematics, vol. 11, no. 5, pp. 940 955, 2013

[26] S. K. Nimbhokar, M. P. Wasadikar, and L. DeMeyer, "Coloring of meet-semilattices," Ars Combinatoria, vol. 84, pp. 97-104, 2007. 




Advances in

Operations Research

mansans

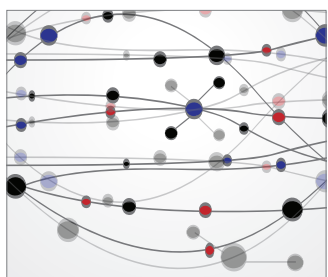

The Scientific World Journal



Submit your manuscripts at http://www.hindawi.com
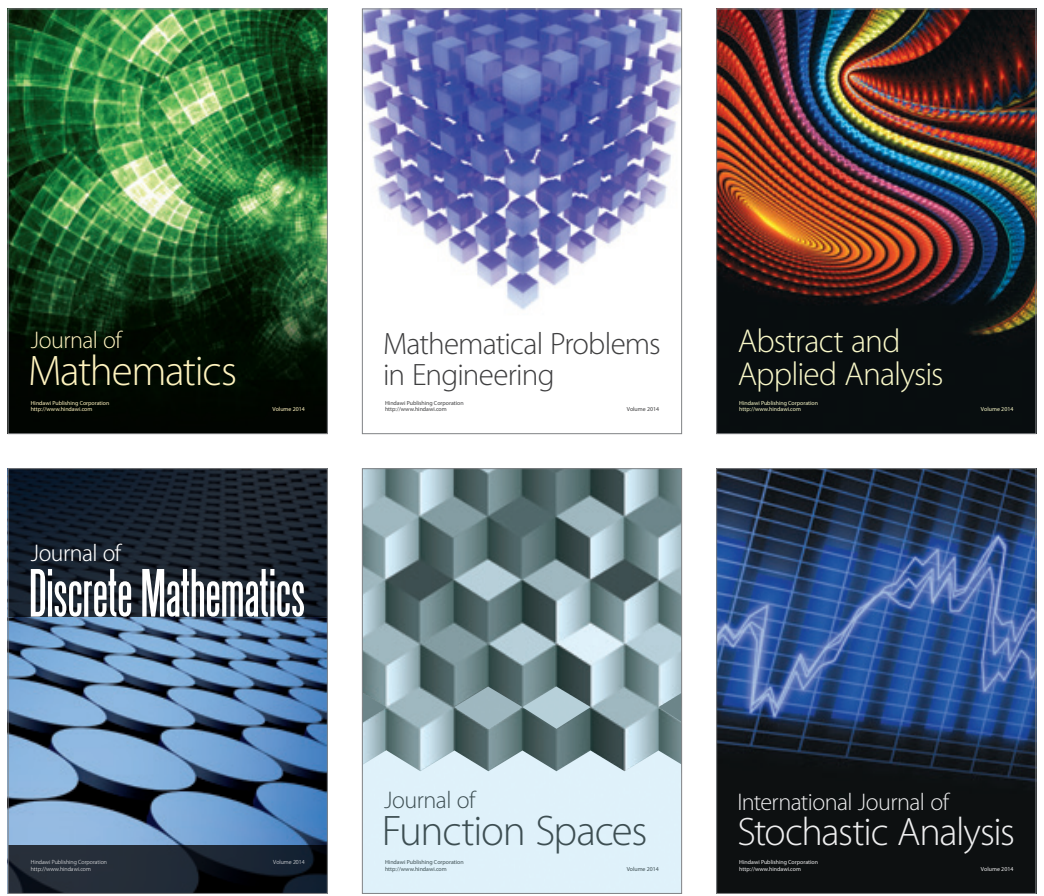

Journal of

Function Spaces

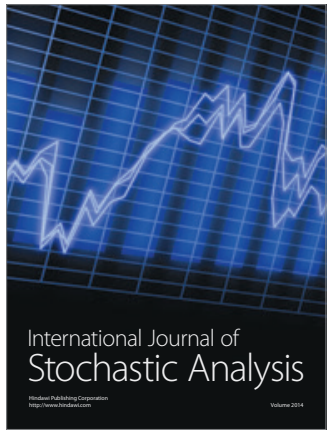


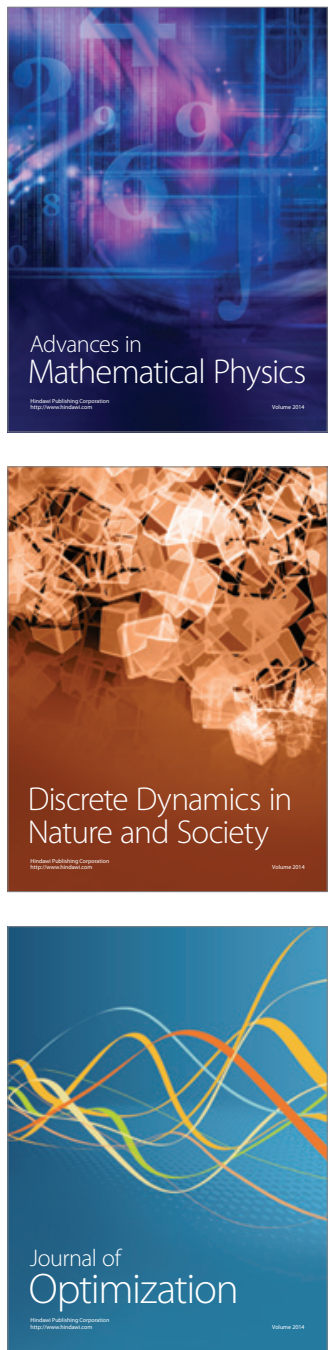